Copyright (C) 2006 IEEE. Reprinted from IEEE Transactions on Geoscience and Remote Sensing, 2004; 42 (9):1803-1810

This material is posted here with permission of the IEEE. Such permission of the IEEE does not in any way imply IEEE endorsement of any of the University of Adelaide's products or services. Internal or personal use of this material is permitted. However, permission to reprint/republish this material for advertising or promotional purposes or for creating new collective works for resale or redistribution must be obtained from the IEEE by writing to pubs-permissions@ieee.org.

By choosing to view this document, you agree to all provisions of the copyright laws protecting it. 


\title{
Development of a Ground-Based Polarimetric Broadband SAR System for Noninvasive Ground-Truth Validation in Vegetation Monitoring
}

\author{
Zheng-Shu Zhou, Wolfgang-Martin Boerner, Fellow, IEEE, and Motoyuki Sato, Senior Member, IEEE
}

\begin{abstract}
We have developed a ground-based polarimetric broadband synthetic aperture radar (SAR) system for noninvasive ground-truth validation in polarimetric SAR remote sensing of terrestrial vegetation cover. This system consists of a vector network analyzer, one dual-polarized antenna, and an antenna positioner. It can be operated in a frequency range from $50 \mathrm{MHz}$ to $20 \mathrm{GHz}$, with a scanning aperture of $20 \mathrm{~m}$ in the horizontal and $1.5 \mathrm{~m}$ in the vertical direction. Tests carried out with standard reflectors showed that the polarimetric measurement capabilities of this system are satisfactory. Using the polarimetric ground-based SAR (GB-SAR) system, we carried out measurements on a specific vegetation cover pertinent to the remote sensing of forested regions within Sendai City, consisting of three different kinds of trees common within the Kawauchi Campus of Tohoku University. Measurements were collected in spring, summer, and autumn. Three-dimensional (3-D) polarization-sensitive images were reconstructed from the acquired data. Analysis of the 3-D polarimetric images of each measurement found differences (at times strong differences) among the polarization signatures. There were stronger reflections in all of the $\mathrm{HH}, \mathrm{VH}, \mathrm{VV}$ images in the second (summer) measurement, especially in the VH image, due to the substantial growth of branches and leaves in summer. This ground-truth validation system provided valuable information about the scattering mechanisms of the three trees selected for analysis in different seasons, which can be detected by broadband polarimetric ground-based SAR measurements. The experimental results demonstrate the excellent polarimetric performance of the newly developed SAR imaging system, which should find many useful and immediate applications in noninvasive ground-truth validation of diverse terrestrial vegetation covers.
\end{abstract}

Index Terms-Polarimetric calibration, radar polarimetry, synthetic aperture radar (SAR), vegetation monitoring.

\section{INTRODUCTION}

$\mathbf{S}$ YNTHETIC aperture radar (SAR) using spaceborne and airborne platforms has attracted interest for many years. Polarimetric SAR (POL-SAR) is a microwave imaging technique

Manuscript received July 8, 2003; revised May 4, 2004. This work was supported in part by the Japan Society for the Promotion of Science under Grant-in-Aid for Scientific Research (S) 14102024.

Z.-S. Zhou is with the Center for Northeast Asian Studies, Tohoku University, Sendai, 980-8576, Japan (e-mail: zszhou@ cneas.tohoku.ac.jp).

W.-M. Boerner is with the Communications, Sensing and Navigation Laboratory, Department of Electrical and Computer Engineering, University of Illinois at Chicago, Chicago, IL 60607-7018 USA (e-mail: wolfgang.m.boerner@ uic.edu).

M. Sato is with the Center for Northeast Asian Studies, Tohoku University, Sendai, 980-8576, Japan (e-mail: sato@ cneas.tohoku.ac.jp).

Digital Object Identifier 10.1109/TGRS.2004.832248 for extracting geophysical texture and volumetric vegetation parameters from SAR images, and its usefulness in terrain classification and surface change detection has been demonstrated [1]. Typical modern methods of SAR polarimetry are radar target decomposition and classification, topographic mapping [1], and more recently, monitoring of ground displacements using differential interferometric and polarimetric ground-penetrating SAR concepts [2], [3]. SAR polarimetry can also be exploited in ground-based radar imaging systems.

Recently, a few design options of ground-based SAR systems have been proposed as monitoring tools for large man-made structures, environmental studies, and ground surface deformation detection. Tarchi et al. [4] showed an application of radar interferometric techniques aimed at monitoring structural deformations of buildings by a ground-based interferometric SAR system. Pieraccini et al. [5] proposed a ground-based interferometric SAR technique for terrain mapping and masonry investigation [6]. Most of these applications of ground-based SAR were based on interferometric techniques and were developed only for narrow bandwidths. In the present work, we have extended those earlier approaches and developed an ultrawideband, ground-based, fully polarimetric SAR system for noninvasive ground-truth validation in environmental studies of terrestrial vegetation cover, and especially in biomass estimation. This work uses the three-dimensional (3-D) imaging algorithm derived from microwave holography as originally introduced by Larson et al. [7], the wave migration technique developed for geophysical exploration [8]-[12], and further two-dimensional (2-D) SAR focusing algorithms discussed in [13]-[16]. A fundamentally more basic origin of the migration algorithm derives from the square-integrable functions of Fourier transforms [17], which have absolute convergence for the square integration implied conservation of energy. The ground-based polarimetric broadband SAR technique has recently been employed by our research group for environmental studies by detecting changes in various kinds of vegetation cover due to seasonal variations. This system makes use of a vector network analyzer, one dual-polarized broadband antenna, and a positioner. By implementing methods of radar polarimetry [2], [18], the ground-based broadband SAR system can be used for monitoring changes in various vegetation and especially tree structure characteristics, such as the growth pattern of leaves and branches due to seasonal variations. In this paper, we describe first a ground-based broadband polarimetric SAR system and 
give the calibration results with some experimental performance examinations, and then interpret experimental results of monitoring seasonal variations in trees. The method provides a most useful tool for noninvasive 3-D ground-truth validation and polarimetric systems calibration of POL-SAR images collected with airborne sensors [the National Aeronautic and Space Administration/Jet Propulsion Laboratory Airborne SAR, the German Aerospace Center E-SAR, the Japan Aerospace Exploration Agency (JAXA)/National Institute of Information and Communication Technology (NiCT) Polarimetric and Interferometric Airborne Synthetic Aperture Radar (Pi-SAR)] and spaceborne (e.g., Space Shuttle Spaceborne Imaging Radar-C/C-Band SAR, the existing Environmental Satellite, and the forthcoming satellites JAXA Advanced Land Observing Satellite (ALOS) Phased Array Type L-Band Synthetic Aperture Radar, Canadian Space Agency RADARSAT-2/CSA) multimodal fully polarimetric POL-SAR and POLinSAR sensors [3].

\section{GROUND-BASEd POLARIMETRIC SAR SYSTEM}

\section{A. System Description}

The radar system consisted of a vector network analyzer, two diagonal dual-polarized broadband horn antennas, an antenna positioner unit, and a personal computer-based control unit. The network analyzer, operated in a stepped frequency continuous-wave mode, was used to generate the transmitting signal and to detect scattered signals both in amplitude and phase. The synthetic aperture was realized by scanning the antennas on a horizontal rail and moving along a vertical post similar to the early microwave holographic image information (hologram) collection systems [7]. The horizontal and vertical scanning aperture widths determine the horizontal and vertical resolutions. The range resolution depends on the radar frequency and bandwidth[19].

Fig. 1 shows our broadband ground-based polarimetric SAR system. This system may operate at frequencies between $400 \mathrm{MHz}$ and $6 \mathrm{GHz}$, with a scanning aperture of $20 \mathrm{~m}$ in the horizontal and $1.5 \mathrm{~m}$ in the vertical range. The working frequency range can however be easily extended from $50 \mathrm{MHz}$ up to $20 \mathrm{GHz}$ when double-ridged waveguide broadband horn antennas and a high-speed extrawideband network analyzer are employed. The measurement and data acquisition were accomplished by a specially designed control program. The 3-D broadband polarimetric radar images can be reconstructed from the scattering data by wave migration algorithms as discussed in [20], which differ somewhat from the microwave holographic image reconstruction methods [7], [13], [16], a topic that deserves further in-depth analyzes. Because a 3-D reflectivity image can be formed by synthesizing the 2-D aperture, a modified 3-D diffraction stacking algorithm was applied to reconstruct the radar image from the scattering data [9]. Based on computer simulation results, some experimental parameters were determined in advance for antenna calibration and system validation.

\section{B. Polarimetric Characteristics of the SAR System}

Several measurements were made for initial characterization of the polarimetric ground-based SAR system. The dual-polarization broadband diagonal horn antenna was calibrated using standard test reflectors. A metallic sphere was used as our first

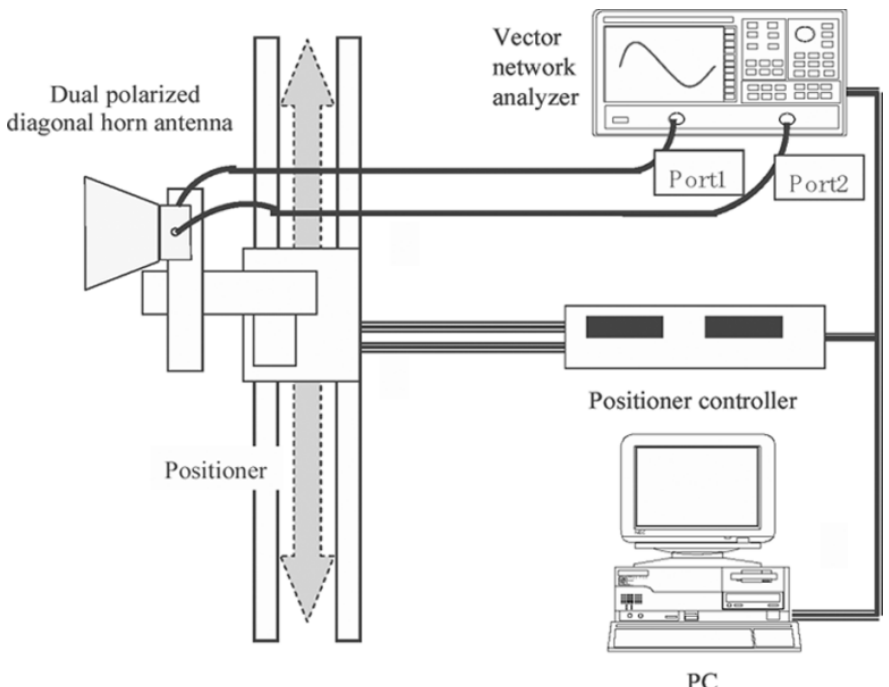

(a)

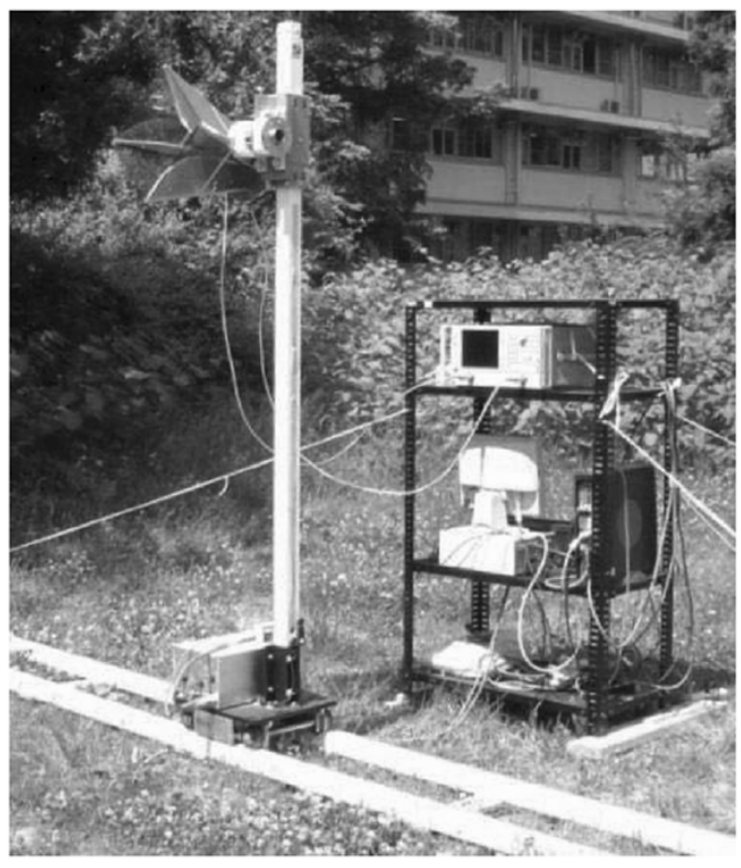

(b)

Fig. 1. Configuration of the broadband ground-based polarimetric SAR system. (a) A schematic and (b) photograph of the developed system as built.

calibration target. If the range distance is not long enough for the dual-polarized diagonal broadband horn antenna for such a wide frequency range from $1-5 \mathrm{GHz}$, the signal returned from the calibration target cannot be distinguished from the directly coupling wave [21]. Another problem was encountered by the long-range separation of the calibration target and its relative electric distance above the ground. Since the calibration target was relatively close to the ground surface, the returned signal could not easily be separated from the inherent ground clutter.

Hence, the arrival time difference between the direct reflection from the target and the wave reflected from the ground and then rereflected by the target must be longer than the continuing reflecting wave (i.e., the arrival time difference between the reflection from the front of the sphere versus the backscatter from the sphere-the creeping wave) from the target. Otherwise, the 


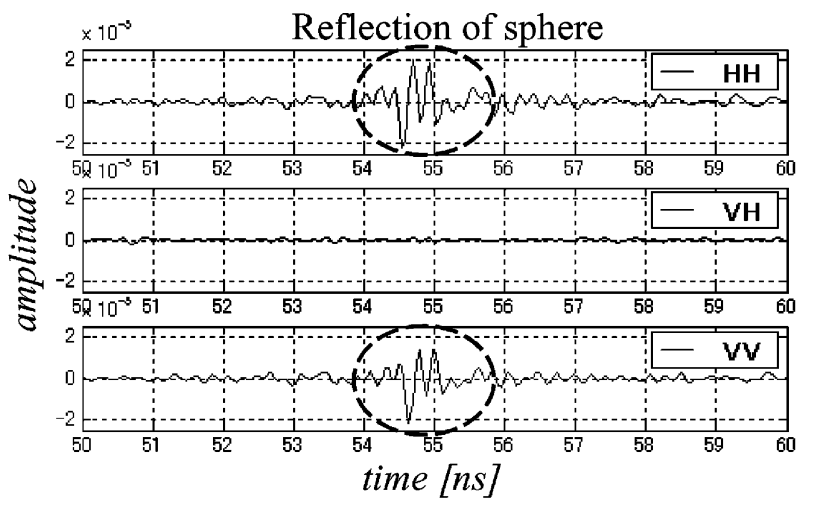

(a)

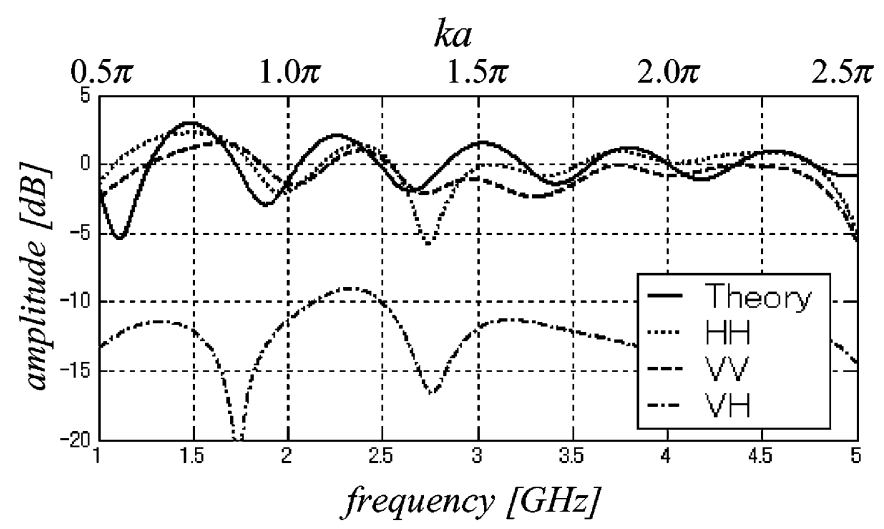

(b)

Fig. 2. Testing scenario with measurement results of a metal sphere with radius $0.075 \mathrm{~m}$. (a) Reflection signal waveforms. (b) Theoretical and measured $\mathrm{HH}$, $\mathrm{VV}$, and VH backscattering curves of the sphere. (Solid line) Theoretical curve. (Dotted line) HH. (Dashed line) VV. (Dashed-dotted line) VH.

reflections of the target and the ground scattering cannot be separated. Fig. 2(a) shows the reflected signal of a metallic sphere of radius $0.075 \mathrm{~m}$. The dashed-line circles indicate reflection from the sphere and the creeping wave contributions.

Substituting the radius of the metallic sphere $a=0.075 \mathrm{~m}$ to (A6) of the Appendix , the equation of normalized radar cross section [22], [23], shown in Appendix A, we obtain the theoretical normalized radar cross section (NRCS) value shown as the solid line in Fig. 2(b). The measured data of $\mathrm{HH}, \mathrm{VV}$, and $\mathrm{VH}$ components compensated by the gain factor of the antenna used in the system are also plotted in Fig. 2(b).

For the case of a sphere, we find that the waveforms of both the HH and VV reflected signals in Fig. 2(a) have very similar amplitude and the same phase. We also observe that there are the same number of peaks and that the resonance points of the measured curves coincide with those of the theoretical value shown in Fig. 2(b). Hence, the measured backscattering data of copolarization components are consistent and very similar to the theoretical value. For our exploratory test set-up, the cross-polarization component is about $12 \mathrm{~dB}$ lower than copolarization components.

Making use of our broadband ground-based polarimetric SAR system, we carried out some experiments with a few of the standard reflectors for radar polarimetry to demonstrate the polarimetric performance of this system. We used a vertical dihedral, a $45^{\circ}$ dihedral, a vertical wire and a $45^{\circ}$ wire as the calibration

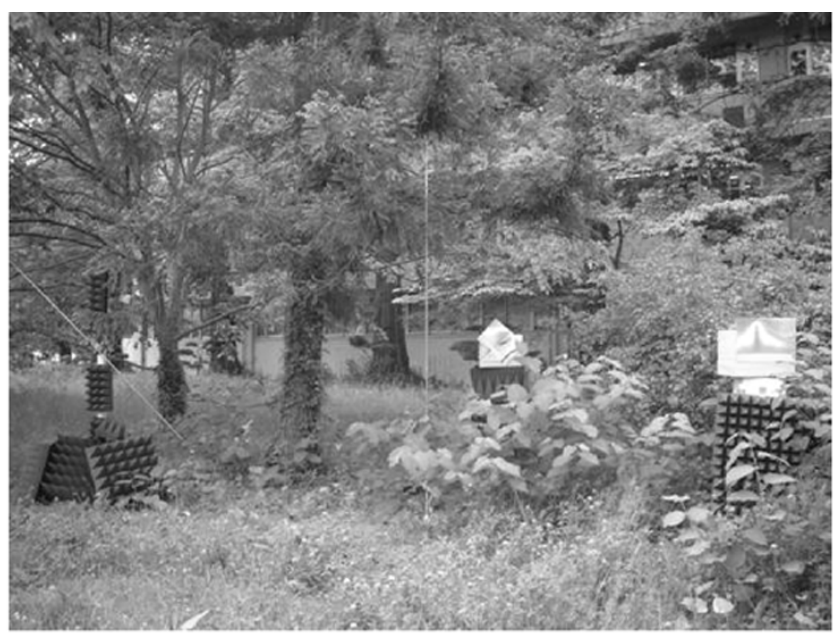

(a)

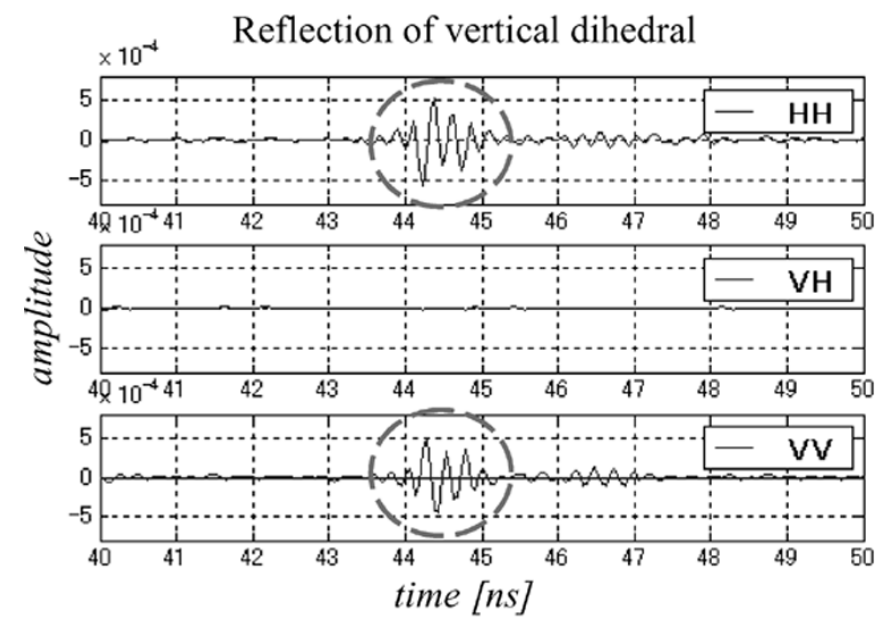

(b)

Fig. 3. (a) Scene with calibrator arrangement and (b) reflected waveforms of a vertical dihedral.

targets shown in Fig. 3(a). According to the reflecting signals of each standard reflector for $\mathrm{HH}, \mathrm{VH}$, and $\mathrm{VV}$ scattering matrix elements, we calculated the associated scattering matrix at various frequencies across the entire spectral measurement domain. The matrix elements for $2 \mathrm{GHz}$ for each reflector are given as follows:

Vertical dihedral:

$S_{m 1}=\left[\begin{array}{ll}0.9813 e^{-j 2 \pi \cdot 0.3158} & 0.0365 e^{j 2 \pi \cdot 0.3583} \\ 0.0365 e^{j 2 \pi \cdot 0.3583} & 1\end{array}\right]$

$45^{\circ}$ dihedral:

$S_{m 2}=\left[\begin{array}{cl}0.2977 e^{-j 2 \pi \cdot 0.3344} & 1 \\ 1 & 0.2891 e^{j 2 \pi \cdot 0.3119}\end{array}\right]$

Vertical wire:

$S_{m 3}=\left[\begin{array}{ll}0.2635 e^{-j 2 \pi \cdot 0.4658} & 0.1345 e^{j 2 \pi \cdot 0.4269} \\ 0.1345 e^{j 2 \pi \cdot 0.4269} & 1\end{array}\right]$

$-45^{\circ}$ wire:

$S_{m 4}=\left[\begin{array}{cl}1 & 0.5747 e^{j 2 \pi \cdot 0.2586} \\ 0.5747 e^{j 2 \pi \cdot 0.2586} & 0.8770 e^{-j 2 \pi \cdot 0.4778}\end{array}\right]$. 
The theoretical scattering matrices of the above four reflectors are known and given respectively by

$$
\begin{array}{ll}
S_{1}=\left[\begin{array}{cc}
-1 & 0 \\
0 & 1
\end{array}\right] & S_{2}=\left[\begin{array}{ll}
0 & 1 \\
1 & 0
\end{array}\right] \\
S_{3}=\left[\begin{array}{ll}
0 & 0 \\
0 & 1
\end{array}\right] & S_{4}=1 / 2\left[\begin{array}{cl}
1 & -1 \\
-1 & 1
\end{array}\right] .
\end{array}
$$

Compared with the theoretical results, good agreement can be found. Moreover, from the $\mathrm{HH}$ and VV reflection waveforms of the vertical dihedral in Fig. 3(b), we observe the same magnitude and inverse phases. These test results demonstrate that the polarimetric performance of our system is more than satisfactory for both narrow and broadband measurements.

A modified polarimetric RCS calibration technique using two orientations of the dihedral corner reflectors as calibration targets was introduced [24]. This method is valid for any monostatic or quasi-monostatic radar system. From the theoretical value of two calibrators and measured data, we recovered the calibration coefficients. Using these calibration coefficients, the calibrated scattering matrices can be calculated. From the calibrated scattering matrices of the data collected from dihedral reflectors given in (2a) and (2b), we find the improvements after calibration to be rather considerable and satisfactory for the intended application, especially in the phase term.

Vertical dihedral:

$$
\begin{aligned}
S_{c 1} & =\left[\begin{array}{ll}
1.0146 e^{-j 2 \pi \cdot 0.4975} & 0.0657 e^{j 2 \pi \cdot 0.2820} \\
0.0657 e^{j 2 \pi \cdot 0.2820} & 1
\end{array}\right] \\
45^{\circ} & \text { dihedral: } \\
S_{c 2} & =\left[\begin{array}{ll}
0.1381 e^{-j 2 \pi \cdot 0.2884} & 1 \\
0.9904 e^{-j 2 \pi \cdot 0.0040} & 0.1285 e^{-j 2 \pi \cdot 0.1200}
\end{array}\right] .
\end{aligned}
$$

\section{Three-Dimensional Imaging}

In order to obtain 3-D images, we used a matched filter for pulse compression [25]. Matched-filter processing of a target echo signal can be thought of as a coherent summation of the reflected signal energy from each of the target's reflection points [26]. Fig. 4 shows an example of pulse compression by the matched filter approach (3), where achievement of the SNR after pulse compression is indicated as follows:

$$
F(\omega)=F_{m}(\omega) \cdot F_{\text {ref }}^{*}(\omega)
$$

where

$$
\begin{array}{ll}
F(\omega) & \text { compressed data; } \\
F_{m}(\omega) & \text { measured data; } \\
F_{\text {ref }}^{*}(\omega) & \text { reference data; and the superscript } * \text { denotes the } \\
& \text { complex conjugate. }
\end{array}
$$

The acquired data are compressed by matched filter compression, in which the reference signal is a reflection from an aluminum plate measured by the same radar system and parameters.

A 3-D SAR image can be reconstructed by using the data set acquired with the two-dimensional aperture by implementing the wave migration method. Wave migrations are often used for image reconstruction by wave field extrapolation, which is similar yet quite distinct from that of microwave holography [7].

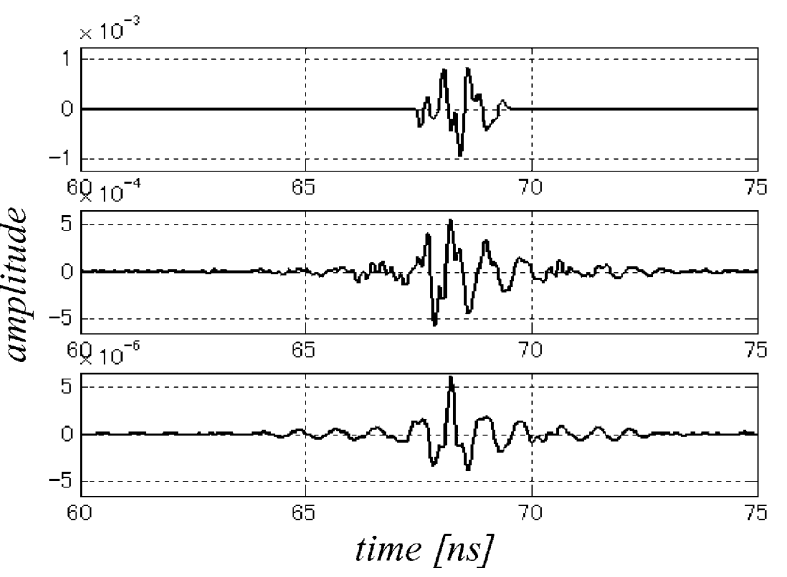

Fig. 4. Pulse compression by matched filter. (a) Reference signal. (b) Raw signal. (c) Compressed signal.

The goal of wave migration is to make the stacked section appear similar to the real location. The diffraction stacking is an integral solution of the wave equation. It can extrapolate wave fields of an observation aperture to larger 3-D space. Diffraction stacking migration produces one migrated sample (a pixel) at a time by computing a diffraction shape for a scatter point at that location, summing and weighting the input energy along a diffraction path, and placing the summed energy at the scatter point location on the migrated section [9], [10].

The origin of this algorithm derives from the square-integrable functions of Fourier transforms. The transformation of migration is a spatially variant many-to-one mapping in $L 2$, where $L 2$ refers to a mathematical space that is square-integrable [17], as in

$$
\int_{-\infty}^{\infty}|f(t)|^{2} d t<\infty
$$

where $f$ is in $L 2$ space. It lies at the heart of the formality in which Fourier transforms behave according to the law of energy conservation etc. This fundamental lemma often is assumed, explicitly or implicitly, as the basis for analysis of many radar systems, including especially SAR, and similar signal and imaging processing systems.

In our case, 2-D scanning data are recorded in the domain of $\left(x_{r}, z_{r}, \omega\right)$, then transformed to the domain of $\left(x_{r}, z_{r}, t\right)$ by inverse fast Fourier transform, where $\left(x_{r}, z_{r}\right)$ is the measuring point. Each trace can be thought as a point source radiating, at time $t=0$, a spherical wave that reaches the sampling point (a pixel of 3-D imaging space) after different time intervals.

The arrival times relative to the same target [echoes relative to the same scatterer $\left(x_{i}, y_{j}, z_{k}\right)$ ], if displayed in the $x-t$ plane, cluster along a curve which is a hyperbola [13] with apex in $x_{0}=x$ (the along azimuth position of the source), $t_{0}=2 y_{j} / v$. For 3-D case, the distribution of cluster echoes should be on the surface of a hyperboloid with apex at $\left(x_{i}, y_{j}, z_{k}\right)$. Therefore, for reconstructing the image of this scatterer, we sum the energy along the diffraction path (the hyperboloid surface), and place the summed energy at the scatter point location on the migrated section $\left(x_{i}, y_{j}, z_{k}\right)$. The process is repeated for all migrated samples and for the full polarimetric data set in order to reconstruct 3-D images. 


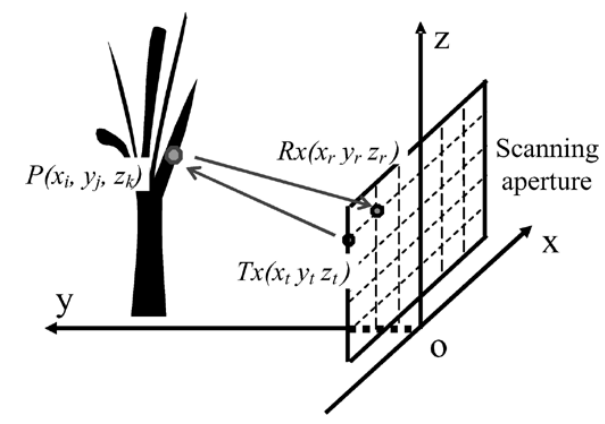

Fig. 5. Illustration of diffraction stacking algorithm.

The 3-D image reconstruction via modified diffraction stacking, derived from a back-projection algorithm [15], [16], is obtained in terms of time delays by

$$
P\left(x_{i}, y_{j}, z_{k}\right)=\iint_{S} f\left(t, x_{r}, z_{r}\right) f_{\text {ref }}^{*}(-t) d z_{r} d x_{r} .
$$

The pulse compression of the matched filter is also shows in (3) in frequency domain. Then we have (6)-(7), shown at the bottom of the page, which is the round-trip delay of the echoed signal for the target at $\left(x_{i}, y_{j}, z_{k}\right)$ when the receiving antenna is at $\left(x_{r}, 0, z_{r}\right) . S$ is the 2-D synthetic aperture, where
$f\left(t, x_{r}, z_{r}\right)$ pulse compressed data in time domain;
$\operatorname{Tx}\left(x_{t}, y_{t}, z_{t}\right)$ transmitter position;
$R x\left(x_{r}, y_{r}, z_{r}\right)$ receiver position;
$P\left(x_{i}, y_{j}, z_{k}\right) \quad$ reconstructed images in spatial domain;
$v \quad$ velocity of electromagnetic wave.

Fig. 5 gives descriptions of these variables.

A migration method of modified diffraction stacking [13], [16] has been employed to reconstruct the complete polarimetric images such that the specific polarization behavior is correctly reconstructed at each point in the 3 -D imaging space. This migration algorithm focuses the time-domain scattering signal on the target in space by computing (6).

\section{ApPlication to TREe Monitoring}

\section{A. Measurements}

We selected three typical local trees as our research objects for which the full polarimetric scattering matrix data takes were collected as described above. These targets were selected for the following reasons:

1) Trees are some of the most important scatterer components in biomass estimation of the terrestrial covers for which "noninvasive ground-truth validation tools" are still not available or in definite need of subtle improvement as regards the implementation of air/spaceborne POL-SAR and POLinSAR imaging, which are rapidly advancing.

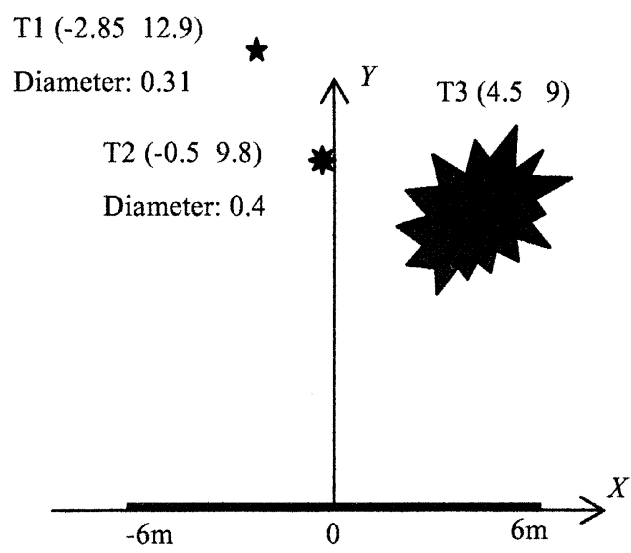

Fig. 6. Coordinate system of measurement site.

2) Different types of trees (deciduous, evergreen, broadleaved, conifer, etc.) have different shapes and substructures to form different scatterers. The implementation of air/spaceborne POL-SAR and POLinSAR imaging allows the best feature classification hitherto available for this type of vegetation cover. However, practical and easily to implement "noninvasive ground-truth validation tools" are lacking.

3) Different trees also change their shapes with different seasons, for example, bare trunk and branches in winter and early spring for most trees and plants, buds in spring, blooming flowers and growth of leaves in late spring and summer, leaves falling off in autumn, and sap contracting into the subsurface root system during winter, and so on. All of these season-dependent factors can best be remotely sensed with air/spaceborne POL-SAR and POLinSAR imaging for which the pertinent "noninvasive ground-truth validation tools" need to be developed most urgently.

4) The tree water content and the moisture of the surrounding air also vary with seasons, and even diurnally during a full day-and-night cycle. That causes at times subtle changes in permittivity, conductivity, and permeability affecting the scattering from these vegetation scatterers. Trees, either alive or dead, display other features which can be detected with air/spaceborne POL-SAR and POLinSAR imaging, again indicating the need to develop "noninvasive ground-truth validation tools."

Using the ground-based polarimetric SAR system described above, which represents such a "noninvasive ground-truth validation tool," data acquisitions were carried out for three different but common types of trees growing near our laboratory;

$$
\begin{aligned}
P\left(x_{i}, y_{j}, z_{k}\right) & =\iint_{S} f\left(t_{i j k}, x_{r}, z_{r}\right) d z_{r} d x_{r} \\
t_{i j k}\left(x_{r}, z_{r}\right) & =\frac{\left(\sqrt{\left(x_{t}-x_{i}\right)^{2}+y_{j}^{2}+\left(z_{t}-z_{k}\right)^{2}}+\sqrt{\left(x_{r}-x_{i}\right)^{2}+y_{j}^{2}+\left(z_{r}-z_{k}\right)^{2}}\right)}{v}
\end{aligned}
$$




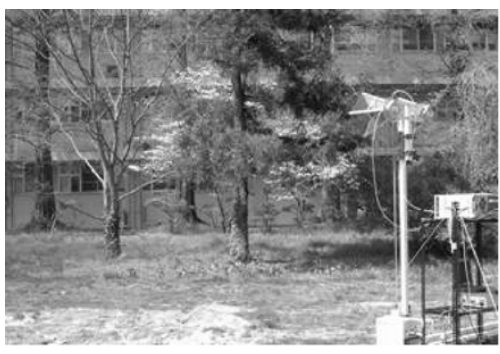

(a) Spring

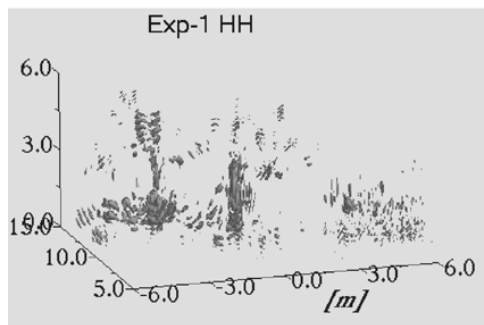

(a1) $\mathrm{HH}$ (amplitude: $5.5 \times 10^{-3}$ )

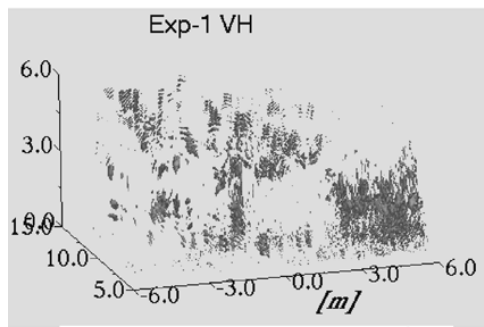

(a2) VH (amplitude: $1.2 \times 10^{-3}$ )

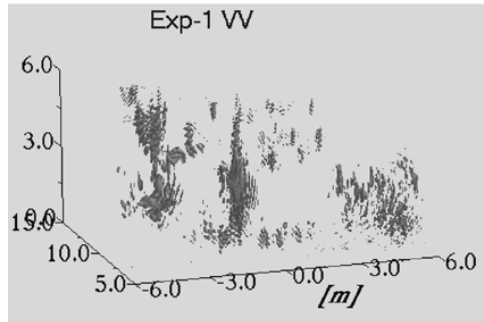

(a3) VV (amplitude: $5.5 \times 10^{-3}$ )

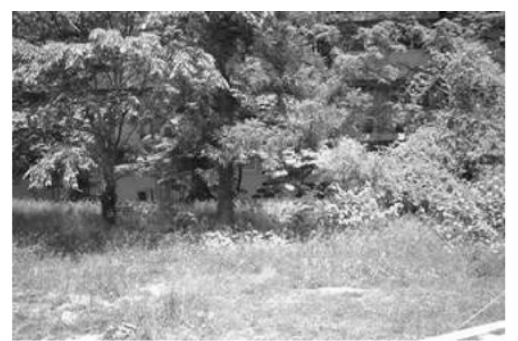

(b) Summer

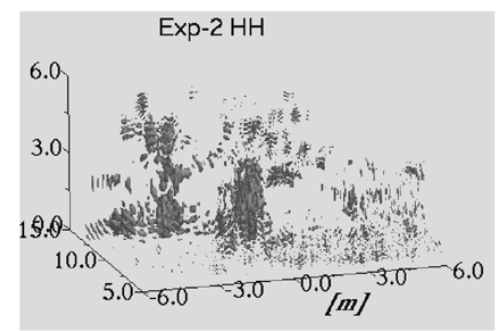

(b1) $\mathrm{HH}$ (amplitude: $5.5 \times 10^{-3}$ )

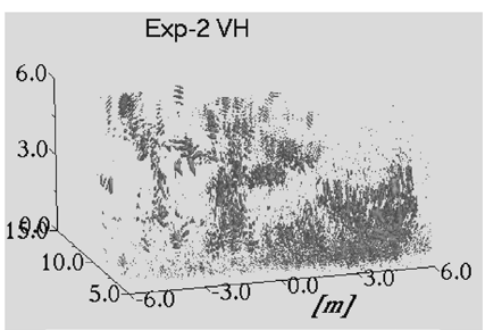

(b2) VH (amplitude: $1.2 \times 10^{-3}$ )

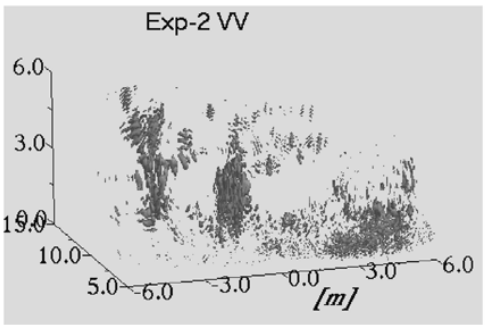

(b3) VV (amplitude: $5.5 \times 10^{-3}$ )

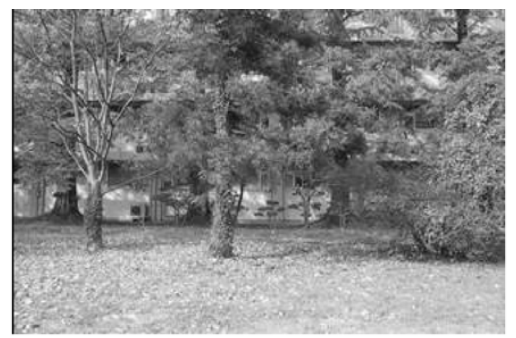

(c) Autumn

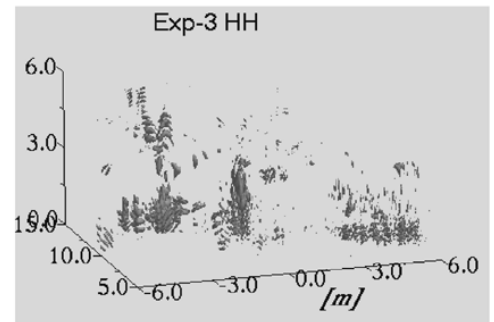

(c1) $\mathrm{HH}$ (amplitude: $5.5 \times 10^{-3}$ )

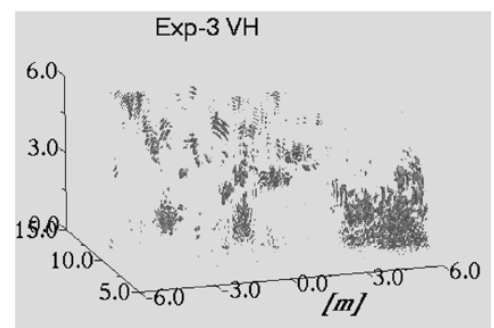

(c2) $\mathrm{VH}$ (amplitude: $1.2 \times 10^{-3}$ )

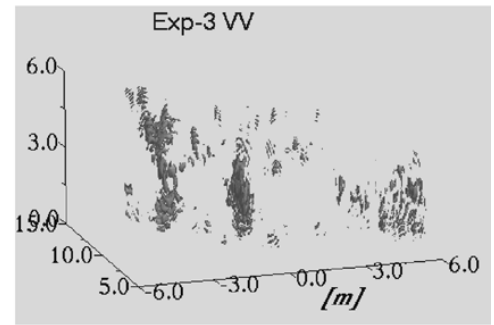

(c3) VV (amplitude: $5.5 \times 10^{-3}$ )

Fig. 7. Scattering scenario with seasonal imaging of different trees. (a) Spring. (b) Summer. (c) Autumn.

measurements were collected over three seasons. The experimental site is located at the Kawauchi Campus, Aoba-Ku of Tohoku University in Sendai, Miyagi-Ken, Japan. The configuration of the target area and of the coordinate system is shown in Fig. 6. The main targets are denoted by T1, T2, and T3, and represent three different kinds of trees: $\mathrm{T} 1$ is a deciduous tree with broad leaves, T2 is an evergreen tree, and T3 is a bush of small height. The three measurements were repeated in spring (April 19, 2002), in summer (May 28, 2002), and in autumn (November 11, 2002), respectively. Fig. 7(a)-(c) shows the experimental scenes in spring, summer, and autumn, respectively. There were a few fresh leaves during the first measurement in spring. Very significant growth in leaves and branches was observed in the second measurement in summer, while the third measurement was at a time when the leaves were falling off.

\section{B. Results and Data Interpretation}

Following pulse compression procedures, the diffraction stacking was applied to migrate the time-domain data in order to acquire the spatial domain data. The 3-D reconstructed images for the $\mathrm{HH}, \mathrm{VH}$, and $\mathrm{VV}$ polarization components of the scattering matrix recovered from the three experiments are respectively shown in Fig. 7. Here the image pixel size is $0.05 \times 0.05 \times 0.05 \mathrm{~m}$. By analyzing the three polarimetric images of each measurement, we can find differences among the different polarizations, which in turn changes considerably from one season to the other depending on the particular tree species, and especially for foliage trees. The $\mathrm{HH}$ image shows the reflections from trunks and some horizontal branches and ground clutter; the VH image indicates strong reflections from leaves and some slant branches; the VV image shows reflections from vertical trunks and branches; and a specifically optimized combination of the scattering matrix components (amplitude and phase included) allows for the distinction between leaves and flowers during the spring season.

Comparing the corresponding images of the different measurements shown in Fig. 7, we find that the reflections from the trunks and the leaves are different and demonstrate seasonal 
variation. There are stronger reflections in the images of Experiment 2 due to the lush growth of branches and leaves in summer. Moreover, the clearer ground surface reflection can readily be seen in Fig. 7(c1)-(c3) in autumn, which agrees well with the real situation. These differences also exist between Fig. 7(a1) and (c1), and between Fig. 7(a3) and (c3). Hence, we can assume that the main reflections were caused by the volume scattering due to the different volume distribution of leaves of different shape and electric properties during the different seasons. Although the shapes of the trees were very similar in late spring and late autumn, the water content of the trees in late spring was higher than that in autumn. This fact caused the slight differences between the reconstructed polarimetric images in late spring and late autumn. The different scattering performances of the different types of trees in the different seasons can be observed clearly demonstrating the intrinsic usefulness of developing our ground-based polarimetric SAR system into a reliable "noninvasive ground-truth validation tool." Additional measurements for a greater variety of the local vegetation structures would be desirable in order to more clearly distinguish the associated scattering mechanisms, which are currently being pursued in collaboration with the arboretum of the Botanic Garden of Tohoku University in Sendai.

\section{CONClusion}

In this paper, the development of a broadband ground-based SAR system and its application to vegetation monitoring are presented with the eventual objective of designing eventually a reliable "noninvasive ground-truth validation tool." Test results showed satisfactory polarimetric performances of the developed system associated with theoretical RCS calculations for a metallic sphere. Calibration was performed using a modified two-way-riented dihedral-based passive polarimetric calibrator. Improvement of the specific polarimetric characteristics of the vegetation images for the broadband ground-based SAR system was achieved by implementing these polarimetric calibration results regularly during the measurement cycle, which usually assumed more than $20 \mathrm{~h}$ for recovering a complete image with our current broadband ground-based SAR system.

Trees represent one of the most important types of vegetation scatterers in studies of local to global-scale biomass estimation. Continuous data acquisitions were carried out for three different common types of trees of the Miyagi prefecture in three seasons at an experimental site close to our laboratory by using a newly developed ground-based polarimetric broadband SAR system. Signal processing methods for the collected broadband ground-based polarimetric SAR data were summarized. It was demonstrated that our algorithms of time gating, matched filtering and diffraction stacking were very effective techniques for our ground-based SAR data processing procedures. They made up for the interference drawbacks of the ground-based imaging system, which included restraining the antenna direct coupling of monostatic returns from bistatic reflections generated by ground clutter, and extrapolating the imaging space by alternate diffraction stacking. The reconstructed 3-D images of $\mathrm{HH}, \mathrm{VH}$, and VV polarization components were consistent with ground-truths and polarimetric radar theory. Hence, radar polarimetry provided valuable scattering information on targets, particularly the scattering features of trees in different seasons, so that the different components of trees contributing to the polarimetric microwave backscatter in different growth phases could be distinguished.

The test results showed that the polarimetric performance of the SAR system is satisfactory and could be implemental for an improved understanding of the underlying scattering mechanisms for widely distributed vegetation scatter as a reliable "noninvasive ground-truth validation tool." It was demonstrated that the broadband ground-based polarimetric SAR system presents advantages for vegetation monitoring as well as for environmental studies of a great variety of vegetation structures, which we are currently further pursuing.

\section{APPENDIX \\ RADAR CROSS SECTION OF SPHERE}

The backscattering radar cross section of a metallic sphere can be calculated from the Mie series by the following formulas [22]:

$$
\sigma=\frac{\lambda^{2}}{\pi}\left|\sum_{n=1}^{\infty}(-1)^{n}(n+0.5)\left(b_{n}-a_{n}\right)\right|^{2}
$$

where

$$
\begin{aligned}
& a_{n}=\frac{j_{n}(k a)}{h_{n}^{(1)}(k a)} \\
& b_{n}=\frac{k a j_{n-1}(k a)-n j_{n}(k a)}{k a h_{n-1}^{(1)}(k a)-n h_{n}^{(1)}(k a)}
\end{aligned}
$$

and

$$
\begin{aligned}
k & =\frac{2 \pi}{\lambda} \\
h_{n}^{(1)}(x) & =j_{n}(x)+j y_{n}(x)
\end{aligned}
$$

where

a radius of sphere;

$\lambda \quad$ wavelength of radar wave;

$h_{n}^{(1)}(x)$ spherical Hankel function of the first kind;

$j_{n}(x) \quad$ spherical Bessel function of the first kind;

$y_{n}(x) \quad$ spherical Bessel function of the second kind.

NRCS is defined as the backscatter factor of a radar target and can be expressed as [23]

$$
\begin{aligned}
\mathrm{NRCS} & =\frac{\sigma}{\pi a^{2}} \\
& =\left(\frac{2}{k a}\right)^{2}\left|\sum_{n=1}^{\infty}(-1)^{n}(n+0.5)\left(b_{n}-a_{n}\right)\right|^{2} .
\end{aligned}
$$

\section{ACKNOWLEDGMENT}

The authors would like to thank the three anonymous reviewers and especially the associate editor for their constructive comments, which helped to improve the quality of this paper. A special note of thanks is extended to R. K. Raney for many thought-provoking suggestions and for providing pertinent references. 


\section{REFERENCES}

[1] W. -M. Boerner et al., "Polarimetry in remote sensing: Basic and applied concepts," in Manual of Remote Sensing, 3rd ed, F. M. Henderson and A. J. Lewis, Eds. $\quad$ New York: Wiley, 1998, vol. 2, ch. 5, pp. 271-358.

[2] S. R. Cloude and K. P. Papathanassiou, "Polarimetric SAR interferometry," IEEE Trans. Geosci. Remote Sensing, vol. 36, pp. 1551-1565, Sept. 1998.

[3] W.-M. Boerner, "Recent advances in extra-wide-band polarimetry, interferometry and polarimetric interferometry in synthetic aperture remote sensing, and its applications," Proc. Inst. Elect. Eng., Radar Sonar Navigat., vol. 150, no. 3, pp. 113-125, June 2003.

[4] D. Tarchi, E. Ohlmer, and A. Sieber, "Monitoring of structural changes by radar interferometry," Res. Nondestruct. Eval., vol. 9, pp. 213-225, 1997.

[5] M. Pieraccini, G. Luzi, and C. Atzeni, "Terrain mapping by groundbased interferometric radar,' IEEE Trans. Geosci. Remote Sensing, vol. 39, pp. 2176-2181, Oct. 2001

[6] D. Tarchi, H. Rudole, M. Pieraccini, and C. Atzeni, "Remote monitoring of buildings using a ground-based SAR: Application to cultural heritage survey," Int. J. Remote Sens., vol. 21, no. 18, pp. 3545-3551, 2000.

[7] R. W. Larson, J. S. Zelenka, and E. L. Johansen, "A microwave hologram radar system,” IEEE Trans. Aerosp. Electron. Syst., vol. AES-8, pp. 208-217, Mar. 1972.

[8] Ö. Yilmaz, Seismic Data Processing. Tulsa, OK: Soc. Exploration Geophys., 1987, pp. 385-426.

[9] J. C. Bancroft, "Seismic imaging: Amplitudes and inversion-Part 1," CSEG Rec., vol. 27, pp. 11-28, Jan. 2002.

[10] D. Loewenthal, L. Lu, R. Roberson, and J. Sherwood, "The wave equation applied to migration," Geophys. Prospect., vol. 24, pp. 381-399, Mar. 1991.

[11] R. Stolt, "Migration by fourier transform techniques," Geophysics, vol. 43, no. 1, pp. 49-76, 1978

[12] J. Gazdag and P. Sguazzero, "Migration of seismic data," Proc. IEEE, vol. 10 , pp. 1302-1315, Oct. 1984.

[13] C. Cafforio, C. Prati, and F. Rocca, "SAR data focusing using seismic migration techniques," IEEE Trans. Aerosp. Electron. Syst., vol. 27, pp. 194-207, Mar. 1991.

[14] R. K. Raney, H. Runge, R. Bamler, I. G. Cumming, and F. H. Wong, "Precision SAR processing using chirp scaling," IEEE Trans. Geosci. Remote Sensing, vol. 32, pp. 786-799, July 1994.

[15] L. M. H. Ulander, H. Hellsten, and G. Stenstrom, "Synthetic-aperture radar processing using fast factorized back-projection," IEEE Trans. Aerosp. Electron. Syst., vol. 39, pp. 760-776, July 2003.

[16] M. Soumekh, Synthetic Aperture Radar Signal Processing. New York: Wiley, 1999, pp. 212-215.

[17] W. M. Brown, Analysis of Linear Time-Variant Systems. New York: McGraw-Hill, 1963, pp. 280-330.

[18] W.-M. Boerner, "Polarization dependence in electromagnetic inverse problems," IEEE Trans. Antenna Propagat., vol. 29, pp. 262-274, Mar. 1981.

[19] H. Rudolf, D. Tarchi, and A. J. Sieber, "Combination of linear and circular SAR for 3-D features," in Proc. IGARSS, Singapore, Aug. 3-8, 1997, pp. 1551-1553.

[20] Z.-S. Zhou, K. Takasawa, and M. Sato, "Interferometric polarimetric synthetic aperture radar system," Proc. SPIE, vol. 4548, pp. 18-23, Oct. 2001.

[21] R. G. Kouyoumjian and L. Perters, "Range requirements in radar crosssection measurements," Proc. IEEE, vol. 53, pp. 920-928, Aug. 1965.

[22] E. F. Knott, Radar Cross Section Measurements. New York: Van Nostrand Reinhold, 1993, pp. 52-195.

[23] J. D. Taylor, Introduction to Ultra-Wideband Radar Systems. Boca Raton, FL: CRC, 1995, pp. 466-468.

[24] J.-R. J. Gau and W. D. Burnside, "New polarimetric calibration technique using a single calibration dihedral," Proc.Inst. Elect. Eng., Microw. Antennas Propagat., vol. 142, no. 1, pp. 19-25, Feb. 1995.

[25] J. C. Curlander and R. N. McDonough, Synthetic Aperture Radar Systems and Signal Processing. New York: Wiley, 1991, pp. 126-152.

[26] D. R. Wehner, High-Resolution Radar, 2nd ed. Norwood, MA: Artech House, 1995, pp. 72-75.

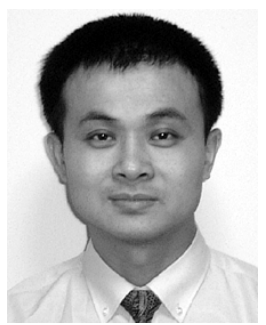

Zheng-Shu Zhou received the B.Sc. degree in physics and the M.E degree in computer science and technology from the Central China Normal University, Wuhan, China, in 1990 and 1999, respectively, and the Dr. Eng. degree in geoscience and technology from the Tohoku University, Sendai, Japan, in 2003.

From 1990 to 1999, he was with the Department of Information Management, Central China Normal University. From 1997 to 1998, he was a Visiting Lecturer with the School of Information and Communications, University of North London, London, U.K. He is currently a Post-Doctoral Research Fellow with the Center for Northeast Asian Studies, Tohoku University. His main research interests include polarimetric radar calibration, broadband radar polarimetry and interferometry, 3-D SAR imaging algorithms, signal and image processing, numerical analysis of electromagnetic scattering, and wave propagation.

Dr. Zhou is a member of the Institute of Electronics, Information and Communication Engineers of Japan.

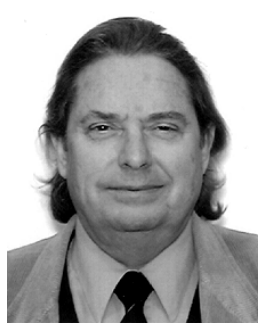

Wolfgang-Martin Boerner (S'66-M'67-SM'75F'84) received the B.S. degree from the Ausust von Platen Gymnasium, Ansbach, Germany, the M.S. degree from the Technical University of Munich, Munich, Germany, and the Ph.D. degree from the Moore School of Electrical Engineering, University of Pennsylvania, Philadelphia, in 1958, 1963, and 1967, respectively.

From 1967 to 1968, he was a Research Assistant Engineer at the Department of Electrical and Computer Engineering, Radiation Laboratory, University of Michigan, Ann Arbor. From 1968 to 1978, he was with the Electrical Engineering Department, University of Manitoba, Winnipeg, MB, Canada. In 1978, he joined the Department of Electrical Engineering and Computer Science, University of Illinois, Chicago, where he is a Professor and Director of Communications and Sensing Laboratory.

Dr. Boerner is a Fellow of the OSA, SPIE, and AAAS. He has been awarded the Alexander von Humboldt U.S. Senior Scientist, the Japan Society for the Promotion of of Science Senior U.S. Scientist, and the U.S. Navy Distinguished Senior Professor. He is the University of Illinois Senior Scholar and and was awarded the Doctor Honoris Causa of Tomsk State University Cluster in Tomsk.

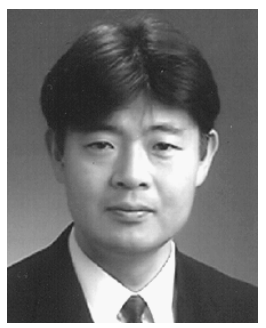

Motoyuki Sato (S'79-M'85-SM'02) received the B.E., M.E., an Dr.Eng. degrees in information engineering in 1980, 1982, and 1985, respectively, all from Tohoku University, Sendai, Japan.

He is currently a Professor at the Center for Northeast Asian Studies, Tohoku University. He joined the Faculty of Engineering, Tohoku University, in 1985. From 1988 to 1989 , he was A Visiting Research with the Federal Institute for Geoscience and Natural Resources (BGR), Hannover, Germany. His current interests include transient electromagnetics and antennas, radar polarimetry, ground-penetrating radar, landmine detection, borehole radar, electromagnetic logging, and interferometric and polarimetrtic synthetic aperture radar.

Dr. Sato has served as Technical Chairman of the 6th International Conference on Ground Penetrating Radar (GPR'96). He was awarded the IEICE (Institute of Electronics, Information, Communication Engineers) Young Engineer's Award in 1984, the SPWLA (Society of Professional Well Log Analysts) Best Poster Award in 1994, and the SEG-J (Society of Exploration Geophysicists-Japan) Best Award in 1999. He is Vice-Chair of the IEEE-GRSS Japan Chapter. 